\title{
Enhanced wavelength-selective absorber for thermal detectors based on metamaterials
}

\author{
Astrit Shoshi $^{1}$, Thomas Maier ${ }^{2}$, and Hubert Brueckl ${ }^{1}$ \\ ${ }^{1}$ Center for Integrated Sensor Systems, Danube University Krems, 2700 Wr. Neustadt, Austria \\ ${ }^{2}$ Molecular Diagnostics, Austrian Institute of Technology GmbH, 1220 Vienna, Austria \\ Correspondence to: Astrit Shoshi (astrit.shoshi@donau-uni.ac.at)
}

Received: 12 October 2015 - Revised: 30 March 2016 - Accepted: 10 April 2016 - Published: 18 May 2016

\begin{abstract}
The dissipative electromagnetic energy absorption of tailored metamaterials can be exploited to improve the spectral sensitivity and selectivity of thermal detectors. The desired detector characteristics are engineered by tuning the single- or multiband absorption by resonance frequency, magnitude, and spectral bandwidth, strongly depending on the geometrical design of metamaterials. Here, the optical absorption properties of trilayer and multilayer resonant structures are investigated by numerical simulations. We consider isotropic, i.e., polarization-independent, disk-shaped absorber elements consisting of alternating aluminium and aluminium nitride layers of nanometer thicknesses, thus representing low-mass absorbers. Trilayer absorbers show spectral resonances at wavelengths between 2 and $6 \mu \mathrm{m}$, reaching near-unity absorption with peak bandwidths ranging from 0.45 to $1.05 \mu \mathrm{m}$. The absorption characteristics remain almost unchanged for radiation with an oblique incidence angle up to $40^{\circ}$. Resonant structures of multilayer absorber elements show besides spectral broadening a dual-band perfect absorption, which are suitable for simultaneous multispectral infrared imaging.
\end{abstract}

\section{Introduction}

Metamaterials are artificial composite structures which exhibit physical properties different from the intrinsic properties of the individual material components (Veselago, 1968). Unusual optical effects such as the negative refractive index (Shelby et al., 2001) and electromagnetic cloaking (Schurig et al., 2006) have been observed, which are hardly accessible in naturally occurring materials (Pimenov et al., 2007). Such properties are derived from the resonant nature of engineered building units with feature size smaller than the wavelength of interest. In general, periodically arranged metaldielectric structures with unit cell dimensions in the subwavelength regime are employed to independently tune the electric (Pendry et al., 1996) and magnetic (Pendry et al., 1999) resonances evoked by the incident radiation. The circulating surface currents induced by plasmon resonance in the metallic layer accompanied by the displacement field in the dielectric layer (Dayal and Ramakrishna, 2012) can be manipulated by changing the geometrical and material parameters. As an effective medium, metamaterials are characterized by homogeneous parameters such as the complex effective electric permittivity $\varepsilon(\omega)=\varepsilon_{1}+i \varepsilon_{2}$ and magnetic permeability $\mu(\omega)=\mu_{1}+i \mu_{2}$ (Smith and Pendry, 2006). In research, much attention has been paid to the real part of $\varepsilon$ and $\mu$ to create materials with negative refractive index, while at the same time minimizing the undesired losses. Similarly, the imaginary loss terms $\varepsilon_{2}$ and $\mu_{2}$ can be engineered to achieve high attenuation and consequently large absorption. By independently manipulating resonances in $\varepsilon$ and $\mu$, it is possible to effectively absorb both the incident electric and magnetic field. Moreover, the impedance of the metamaterial, $Z=\sqrt{\varepsilon(\omega) / \mu(\omega)}$, can be matched to free space, giving rise to minimized reflectivity (Landy et al., 2008). The perfect metamaterial absorber (PMA) is defined to have absorption near unity.

The enhanced absorption properties of metamaterials can be exploited to tailor the spectral responsivity and selectivity of thermal sensors (Landy et al., 2008; Maier and Brueckl, 2009, 2010). Thermal sensors such as bolometer, thermopile, and pyroelectric sensors convert temperature changes caused by the absorption of incident radiation into an electrical signal. They usually have a broad spectral response. Exchange- 
able or fixed optical filter units are used for wavelength selection. In order to achieve a high sensor responsivity, a high absorption is required. Therefore, metamaterials directly integrated on top of thermal sensors are wavelength-selective with an efficient absorption and optimized heat energy transfer (Maier and Brueckl, 2009, 2010). With the integration of low-mass metamaterials, more compact and miniaturized thermal devices can be designed without significantly affecting their response time.

This study focuses on tailoring the optical properties and composition of micron-sized metamaterial structures based on the requirements arising from their integration as wavelength-selective PMAs in thermal sensors.

\section{Materials and simulation model}

As composite dielectric/metallic materials, highly conductive aluminium (Al) with low ohmic losses and nondissipative aluminium nitride (AlN) dielectric interlayer were chosen. $\mathrm{Al}$ is a good thermal and electrical conductor. AlN has superior thermal and mechanical properties in the infrared. In particular, the high thermal conductivity of AlN is comparable to metals such as $\mathrm{Al}$ and is compared to Al oxide (Zhao et al., 2004) about 10 times higher. This is beneficial regarding the heat transfer efficiency to the energy transducer in an integrated sensor. Moreover, these materials are compatible with standard microelectronic production processes.

A simple layout of a near-unity absorber mitigates lithographic demands (Fig. 1). The disc-like top metallic layer (resonator) of an Al-AlN-Al trilayer is located in the center of the square-shaped unit cell consisting of the bottom metal and the dielectric layer of side length $p$. Due to the circular resonator shape, the excitation of the resonance is expected to be isotropic and, thus, independent of radiation polarization (Dayal and Ramakrishna, 2012). As will be discussed later, the trilayer absorber is additionally extended to a multilayer absorber with alternating dielectric/metallic stacks (Fig. 1). Moreover, a passivation layer, which is usually found in thermal sensors - e.g., $\mathrm{SiO}_{2}$ in thermopiles, $\mathrm{Si}_{3} \mathrm{~N}_{x}$ in bolometers, or Au in pyroelectric detectors - is also considered in the layout (Fig. 1). The disk radius in the unit cell determines the areal density of the metamaterial.

Finite-element simulations based on the commercially available software package COMSOL Multiphysics were performed to analyze the optical properties of a reduced three-dimensional model (Fig. 1). The incident light is an electromagnetic plane wave propagating at normal incidence along the negative $z$ direction. The boundary conditions for the unit cell outer walls perpendicular and parallel to the electric field are a perfect electric and perfect magnetic conductor, respectively. For the top and bottom outer walls scattering boundary conditions with and without an incident wave are used, respectively. The experimental refractive index val-

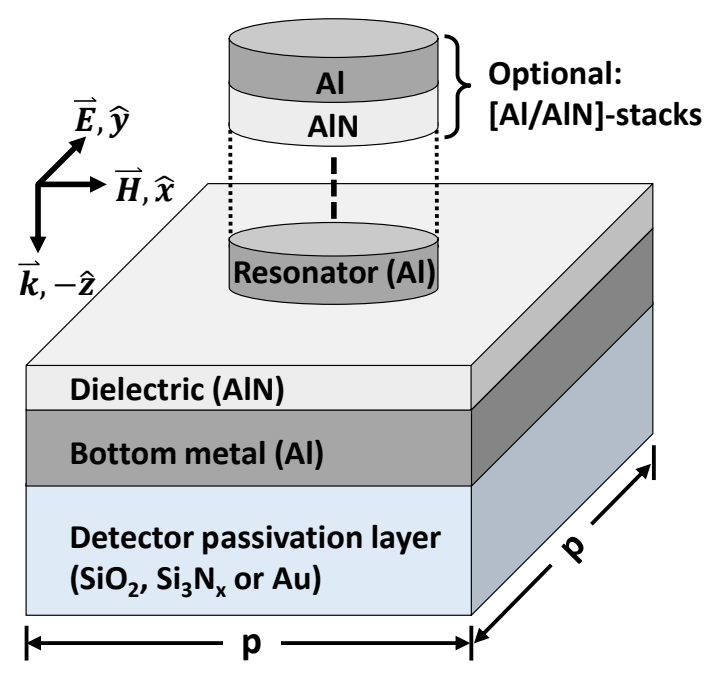

Figure 1. Schematic illustration of the unit cell in the numerical simulations.

ues of the individual materials including the dispersion relation are taken from Kischkat et al. (2012), Ordal et al. (1985), and Palik (1985) and implemented in the model. The field distributions, and the time-averaged power flow in the structure is calculated. Reflection is determined by simulating the same elementary cell geometry with all layer domain properties set to vacuum and normalized to the incident intensity. The difference in the integrated power flow at the individual layer boundaries reveal the absorption values as $A(\omega)=1-R(\omega)-T(\omega)$, with the frequency-dependent reflectance $R(\omega)$ and transmittance $T(\omega)$.

\section{Simulation results}

\subsection{Electric and magnetic resonances}

The incident electromagnetic plane wave propagating in negative $z$ direction excites plasmon resonances in the top metallic layer, and thus polarization currents depending on the electrical permittivity appear inside the metal. The resonator layer at the top behaves like an electric dipole that serves as a coupler to the electric field of the incident wave. Consequently, the electromagnetic fields are concentrated within sub-wavelength regimes leading to a significant increase of the local field strengths (Fig. 2a). The origins of magnetic resonances are antiparallel currents in the metallic layers, which, together with the displacement field in the dielectric layer, result in circulating currents (Dayal and Ramakrishna, 2012; Tao et al., 2008). The current loop induces a magnetic dipole moment that can resonantly couple to the magnetic field vector of the incident light. For a strong localization of the electromagnetic energy within the metamaterial, both an electric and a magnetic dipole resonant coupling at the same frequency are necessary. This resonant coupling is of destructive nature for the reflected direction, thus eliminating reflec- 
tion. In a PMA, neither reflections nor transmissions of the incident light at a given wavelength can be observed (Pendry et al., 1999; Zeng et al., 2013).

The simulated electromagnetic field distributions within the absorber element where both resonances appear are exemplarily shown in Fig. 2. Here, typical geometric parameters for resonator radius and thickness, dielectric, bottom metal, and $\mathrm{SiO}_{2}$ substrate thicknesses are 500 and 50, 110, 150 , and $250 \mathrm{~nm}$, respectively. The distribution of the electric field in Fig. 2a shows the typical dipole excitation and spatial localization within the dielectric interlayer. The concentration of the magnetic field in the dielectric layer demonstrates the confinement of the magnetic field caused by the oscillating current loop (Fig. 2b). The inhomogeneous distribution of the current density in both metal layers is depicted in Fig. 2c. For smaller wavelengths in close proximity to the resonance, the surface current density displays a rather homogeneous distribution with significantly lower current magnitudes. By approaching the resonance wavelength, the current density experiences a progressive increase at the outer center of the resonator and reaches its maximum value at resonance wavelength. For wavelengths beyond the resonance, the current density steadily decreases. It is noteworthy that the surface current reverses its sign from positive to negative, resulting in a directional change of the circulating current loop. The normalized current density magnitude is slightly asymmetric for wavelengths smaller and larger than the resonance wavelength. The induced image charges in the bottom metal layer behave similar to those observed in the top metal layer. The current sheets in the bottom layer are antiparallel oriented and show slightly lower amplitudes compared to the top layer. In agreement to theoretical predictions, the current sheets in both metallic layers form a circulating current loop, have comparable amplitudes, and are slightly out of phase (Dayal and Ramakrishna, 2012; Zeng et al., 2013). For wavelengths far from the resonance, the whole resonant structure acts like an ordinary material with a current density behavior according to its intrinsic characteristics. Figure $2 \mathrm{~d}$ displays the corresponding time-averaged energy flow of the electromagnetic wave in the absorber element at resonance. The energy flows from the outer border towards the center of the resonator. The energy flow in minus $z$ direction averaged over the entire unit cell volume demonstrates the wavelengthdependent energy transfer. At resonance wavelength, an enhancement energy transfer by a factor of about 25 is observed (Fig. 2e).

\subsection{Trilayer absorbers at normal light incidence}

The absorption behavior of circular-shaped trilayer resonators with regard to their geometrical design is discussed. The angle of radiation incidence is $90^{\circ}$, i.e., normal incidence. First, the influence on the optical properties by varying the lateral dimensions as well as the layer thicknesses of the resonator, dielectric, and the bottom metal layer are
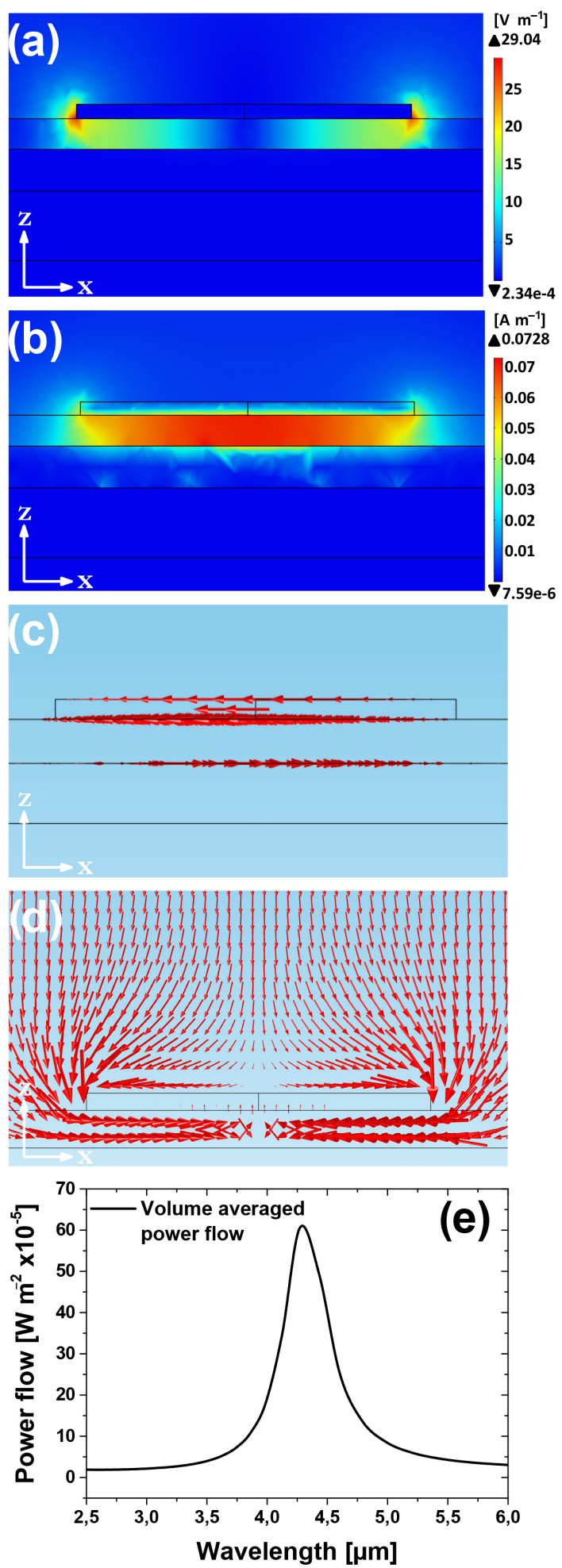

Figure 2. Optical properties of metamaterials for simultaneous electric and magnetic resonances. (a) Normalized electric field and (b) magnetic field. (c) Surface current density. (d) Time-averaged energy flow defined by the Poynting vector. (e) Volume-averaged energy flow ( $z$ component) over the spectral range of interest. 
presented. Moreover, the effects arising from changes in the substrate layer thickness and material as well as the resonator density or filling factor are discussed.

Figure 3a shows a selection of absorption spectra for resonator radii varying between 275 and $600 \mathrm{~nm}$, while all other parameters were kept constant. The layer thicknesses of the resonator, dielectric, bottom metal, and $\mathrm{SiO}_{2}$ substrate are 50, 115,50 , and $250 \mathrm{~nm}$, respectively. The unit cell side length is $2.1 \mu \mathrm{m}$. A variation of the resonator radius results in a clear shift of the resonance peak, which indicates a change in the effective dielectric permittivity of the metamaterial structure. The resonance wavelength experiences a linear red shift with increasing radius. According to a linear fit, a radius change of $10 \mathrm{~nm}$ causes a shift in the resonance wavelength of $80 \mathrm{~nm}$. This is a good reference value in order to estimate peak shifts due to variation in the size of the absorber element, i.e., geometric tolerances caused by fabrication errors. The full width at half maximum (FWHM) of the absorption peak gives information about the coupling strength of excited oscillations in the metamaterial system. There is a linear dependence on the resonator radius ranging from 275 to $600 \mathrm{~nm}$ with FWHM values of 100 and $700 \mathrm{~nm}$, respectively. The observed peak broadening is due to a gradual increase of dissipative losses in the metamaterial.

Figure $3 \mathrm{~b}$ presents the calculated absorption properties for varying dielectric thicknesses between 30 and $210 \mathrm{~nm}$, while all other parameters remain constant: resonator radius and height, bottom metal and substrate thicknesses, and unit cell length are 525 and $50 \mathrm{~nm}, 50$ and $250 \mathrm{~nm}$, and $2.1 \mu \mathrm{m}$, respectively. A change in the dielectric thickness influences primarily the inductance or magnetic resonance and, thus, the effective magnetic permeability of the absorber system. A varying dielectric thickness also affects the capacitive coupling to the bottom metal layer, i.e., it changes the effective permittivity, but in a less pronounced manner. Basically, lower layer thicknesses result in higher capacity and lower inductance of the system and vice versa. The resonance frequency remains constant at a resonance wavelength of $4.5 \mu \mathrm{m}$ in a broad thickness range of 70-210 $\mathrm{nm}$ (Fig. 3b). This behavior reflects the relation of the resonance frequency $\left(\omega_{0}\right)$ to the capacitance $(C)$ and inductance $(L)$ of the structure, which can be considered as an undamped resonant circuit:

$\omega_{0}=1 \sqrt{L C}$.

For dielectric thicknesses below $70 \mathrm{~nm}$, there is an imbalance between the inductance and capacitance of the structure, which results in a significant red shift of the resonance along with a reduction in the amplitude. This behavior is characteristic for a damped oscillation. Figuratively speaking, a steady decrease of the dielectric layer thickness means a gradual approach of the antiparallel-oriented magnetic fields in the resonator and bottom metal layer generated by the two corresponding antiparallel-circulating current loops. For large dielectric thicknesses, an antiparallel orientation of the magnetic fields is energetically favored. A steady decrease of
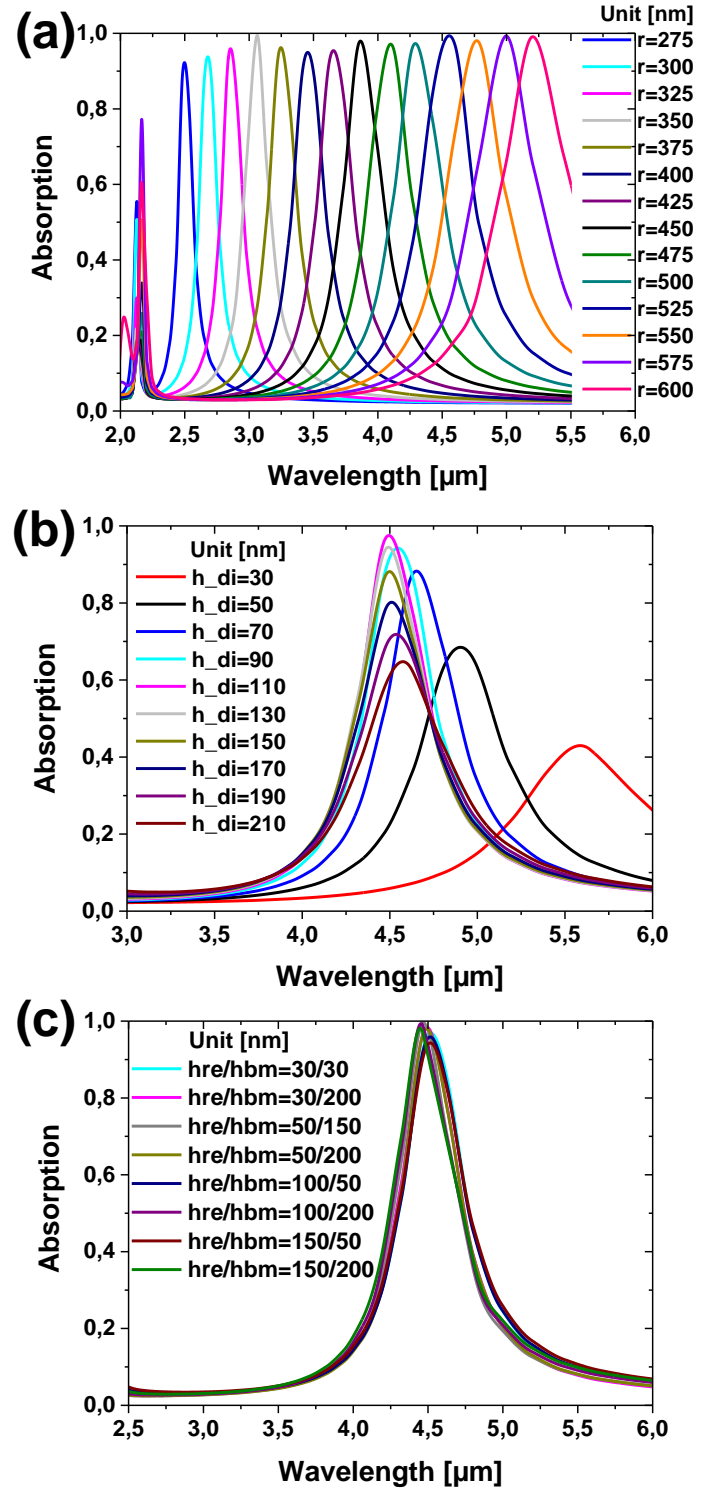

Figure 3. Numerical calculations of absorption spectra for varying (a) resonator radius, (b) dielectric layer thickness, and (c) resonator (hre) and bottom metal (hbm) thickness.

the dielectric layer leads to an increased perturbative interaction. As a consequence, the magnetic fields could perform precession motions when the dielectric thickness falls below a critical value $(\sim 70 \mathrm{~nm})$. A precession means a distortion of flow of the current loops compared to the case for larger dielectric thicknesses. A change in the current flow can be considered as an additional inductance source. The total inductance ( $L=L_{\mathrm{g}}+L_{\mathrm{p}}$ ) of the structure is then the result of the geometrical inductance $\left(L_{\mathrm{g}}\right)$ and the inductance induced by current perturbations $\left(L_{\mathrm{p}}\right)$. Due to the additional perturbative losses, the magnetic resonance frequency does not solely scale up with the geometrical inductance $\left(L_{\mathrm{g}}\right)$. By introducing an effective damping factor to Eq. (1), both a shift of the 
resonance frequency and a reduction of the amplitude can be explained. However, at very thin dielectric thicknesses, the direction of the magnetic field of the bottom metal layer flips and aligns parallel to the upper resonator magnetic field due to energy minimization reasons. In this configuration no magnetic resonances occur, and the absorption properties are determined by the materials permittivity. The calculations show that the amplitude reaches its maximum value of 0.96 at a dielectric thickness of $110 \mathrm{~nm}$ and decreases for larger and smaller layer thickness values similar to a negative parabolic function. The absorption remains within the range 90-130 nm around 0.9. The FWHM value of about $475 \mathrm{~nm}$ is lowest at a thickness of $110 \mathrm{~nm}$, and it remains within the range of 90-130 nm almost constant. Smaller or larger layer thicknesses result in a FWHM increase up to $950 \mathrm{~nm}$.

In a PMA, the layer thickness of both the resonator and the bottom metal needs optimization. The penetration depth of an electromagnetic wave into a metallic layer and, thus, the transmission $T(\omega)$ is determined by the wavelengthdependent skin effect. If the thickness of the bottom layer is smaller than the skin depth, image charges are not effectively formed in the bottom metal layer, deteriorating the magnetic resonance. This also implies a dielectric dipole resonance without a proper impedance matching. Therefore, an effective energy transfer is hindered, and unwanted reflections might occur (Dayal and Ramakrishna, 2012). The resonator (bottom layer) thickness range of 30 to $150 \mathrm{~nm}$ (30 to $200 \mathrm{~nm}$ ) was analyzed. The results shown in Fig. 3c are calculated for $525 \mathrm{~nm}$ radius, $110 \mathrm{~nm}$ dielectric height, $250 \mathrm{~nm}$ substrate height, and $2.1 \mu \mathrm{m}$ unit cell length. For all investigated thickness permutations, the absorption remains always larger than 0.9 and reaches the near-unity absorption value of 0.995 for the pair combination of $50 \mathrm{~nm}$ resonator thickness and $150 \mathrm{~nm}$ bottom metal thickness. The position of the resonance peak remains constant for all combinations. The FWHM has a slightly oscillatory behavior within the range of $495 \pm 25 \mathrm{~nm}$.

Thermal detectors are capped for instance by insulating layers such as $\mathrm{SiO}_{2}, \mathrm{Si}_{3} \mathrm{~N}_{x}$, or conductive Au layers. Their influence on the absorption of integrated metamaterials is also investigated in the simulation model (Fig. 1). In all simulations up to here, a $\mathrm{SiO}_{2}$ substrate of $250 \mathrm{~nm}$ was assumed. $\mathrm{SiO}_{2}$ thickness variations up to $3 \mu \mathrm{m}$ show no noticeable changes in the absorption behavior (data not shown). In the case of a gold passivation layer variation (30 to $200 \mathrm{~nm}$ ), a slightly decreased absorption within the range of 50 to $110 \mathrm{~nm}$ from near unity to 0.95 could be observed. Since the transmission $T(\omega)$ in this metamaterial structure is negligible, the marginal absorption deviations can be explained by changes in the image charge formation in the bottom layer and energy flow direction affected by the conductive Au layer.

The resonator density or filling ratio, i.e., resonator base surface area compared to the elementary cell base surface area, affects the absorption. A resonator density of more

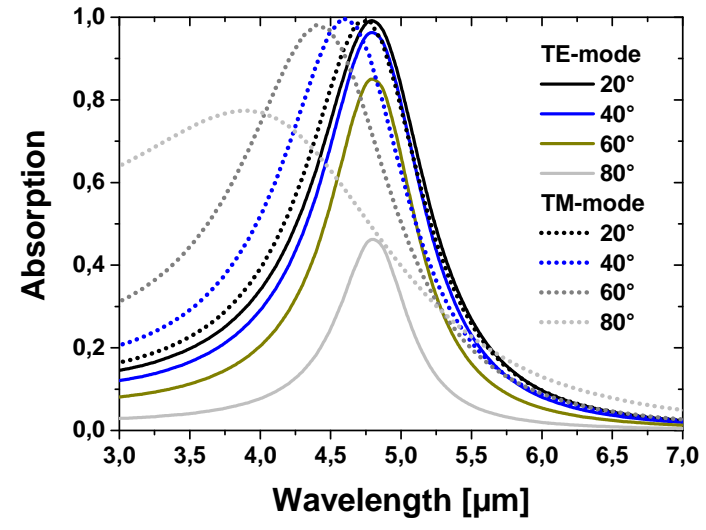

Figure 4. Absorption properties for TE and TM polarization at oblique angle of light incidence.

than $50 \%$ is required for high absorption values, while a decreasing absorption is observed for smaller values (data not shown). Similar to our previous studies (Maier and Brueckl, $2009,2010)$, there is no indication of coupling between the absorber elements down to a lateral absorber-to-absorber distance of $600 \mathrm{~nm}$. Thus, for distances larger than $600 \mathrm{~nm}$ no influence on the resonance frequency could be observed. Due to the short-range nature of the lateral field distribution at resonant frequency (Fig. 2a and b), coupling phenomena occur typically at distances of about half the resonator diameter.

\subsection{Trilayer absorbers at oblique light incidence}

Conventional thermal detectors usually respond to a broad spectral range of the incident radiation. A wavelengthselective response is achieved by implementing exchangeable optical filters or microfilters in front of the detector. Such filters possess an angular transmission characteristic and show a limited selective performance above a critical angle of incidence. Here, the angular dependence of the absorption of metamaterial structures are simulated based on the transmission line model. Similar geometric parameters are chosen: the individual thicknesses of the resonator, dielectric, bottom metal are 50,110, and $150 \mathrm{~nm}$, respectively, with a resonator radius of $500 \mathrm{~nm}$.

For transverse-electric (TE) or transverse-magnetic (TM) polarized light at normal incidence, both the electric and magnetic field are aligned parallel to the resonator plane and are represented by the corresponding in-plane field components. If we consider the case of TE or TM polarization at oblique light incidence, the respective in-plane component of the electric or magnetic field remain unchanged, while the respective in-plane magnetic $\left(H_{\text {ip }}\right)$ or electric $\left(E_{\text {ip }}\right)$ field component parallel to the resonator plane decrease with incidence angle $\alpha$ as $H_{\text {ip }}=H_{0} \cos (\alpha)$ and $E_{\text {ip }}=E_{0} \cos (\alpha)$, with $H_{0}$ and $E_{0}$ being the corresponding field magnitudes at normal incidence. In contrast to normal light incidence, we obtain at oblique radiation incidence additional out-of- 

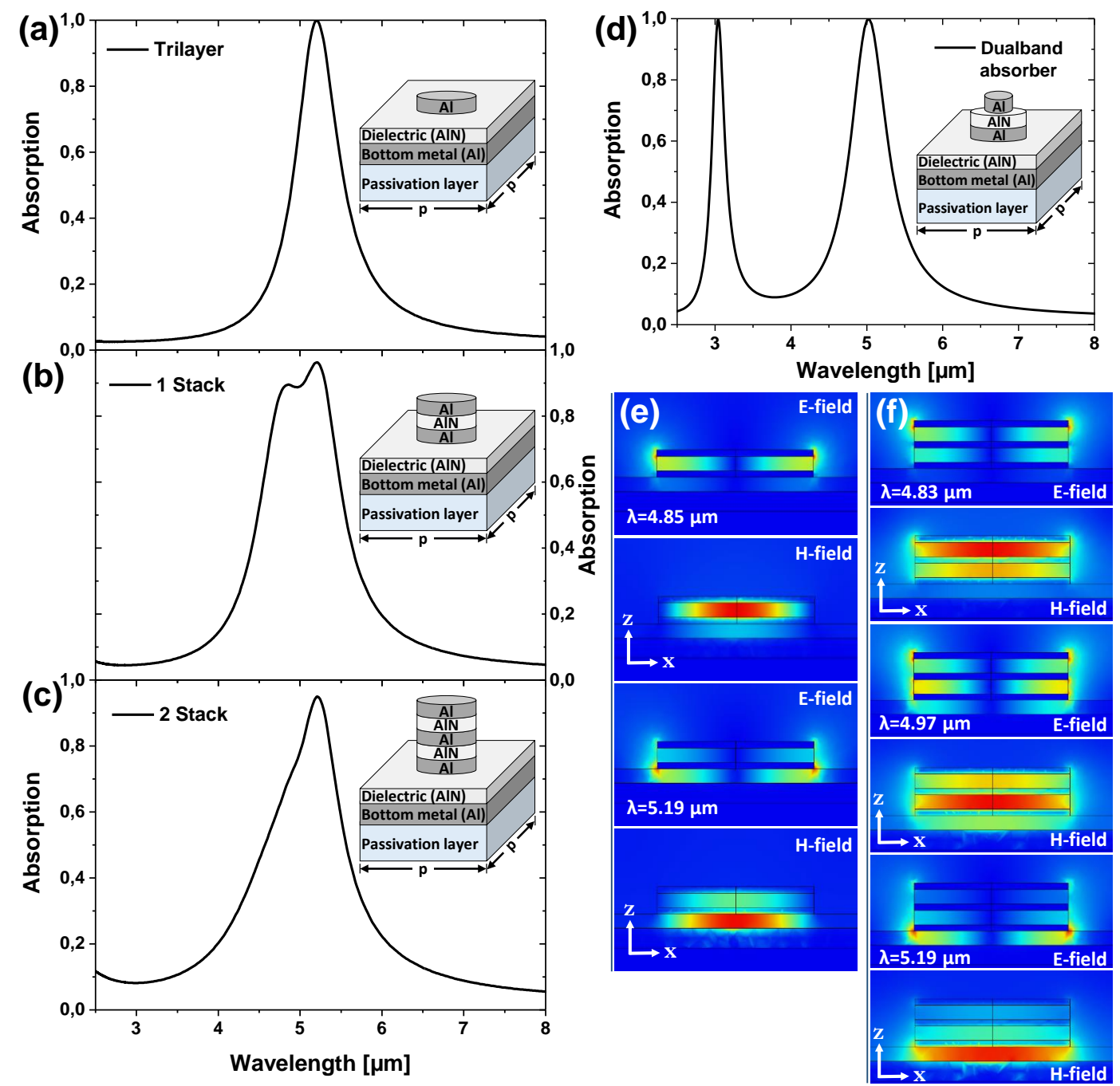

Figure 5. Multilayer absorption spectra for (a) trilayer, (b) one-stack, and (c) two-stack systems. (d) Dual-band absorber based on a modified one-stack absorber. All insets show the specific stack configurations. (e) and (f) represent the electric and magnetic field distribution in the absorber at different resonance wavelengths related to absorption spectra shown in (b) and (c), respectively.

plane field components $\left(H_{\mathrm{op}}, E_{\mathrm{op}}\right)$ perpendicular to the resonator plane, which increase with incidence angle according to $H_{\mathrm{op}}\left(E_{\mathrm{op}}\right)=H_{0}\left(E_{0}\right) \sin (\alpha)$. Figure 4 illustrates the angular dependence of the TE and TM mode of the incident radiation with the electric and magnetic field aligned parallel to the long semi-axis, respectively. For both TE and TM polarized light the absorption remains higher than 0.94 , and a slight shift in the resonance wavelength can be observed up to an angle of incidence of $40^{\circ}$. For the TE mode even $85 \%$ of the intensity is absorbed at an angle of $60^{\circ}$. The overall nonlinear decrease reaches $45 \%$ absorption at $80^{\circ}$. Remarkable for the TE mode is an unchanged resonance wavelength for all angles with a steady nonlinear decrease in the absorption. Characteristic for the TM mode is the high (>90\%) absorption up to an angle of $60^{\circ}$. With rising angle, a slight decrease in the intensity of absorption is observed accompa- nied by a resonance shift of $11 \mathrm{~nm} /{ }^{\circ}$ to smaller wavelengths. Due to Eq. (1), a shift in the resonance wavelength is expected when the inductance and/or capacitance of the systems changes. A gradual angle increase is comparable to a tilt of the resonant structure relative to the incident direction of the electromagnetic wave, and the initial circular shape of the resonator gradually becomes elliptical. This shape transformation from circular to elliptical leads to a decrease of the resonator surface area with increasing angle of incidence. This in turn effectively reduces the capacitance of the resonator which results according to Eq. (1) in a shift of the resonance to smaller wavelengths. However, this implies for TE (TM) polarization a higher impact on the magnetic (electric) resonances, depending on whether the electric or magnetic field is aligned parallel to the resonator plane at all angles. In case of the TE mode, the constant remaining in-plane elec- 
tric field ensures even at high angles of incidence a continual resonant coupling and, thus, plasmon excitation, while the steadily decreasing in-plane magnetic field leads to a gradual magnetic decoupling. As a consequence, the resonance frequency remains constant, while the amplitude decreases nonlinearly with increasing angle of incidence due to magnetic losses. In contrast to the TM mode, the electric losses caused by a successive decoupling of the in-plane electric field with the angle of incidence result in a nonlinear peak broadening and decrease in absorption (Fig. 4). These findings are also supported by an additional simplified simulation approach considering anisotropic resonators at normal light incidence (data not shown). In this approach, a modulation of the shape from initially circular to elliptical was investigated. The short semi-axis is varied, while the long semiaxis remains constant and corresponds to the initial radius. A linear decrease of the short semi-axis with the electric field oriented parallel to it results in a clear linear resonance shift to smaller wavelengths, while the amplitude remains almost unchanged. For the antiparallel-oriented electric field, a clear amplitude decrease without significant alteration of the resonance frequency is observed. Although the results of the two approaches are not one-to-one transferable, they still share some common basic properties and help to qualitatively interpret results or to recognize tendencies.

\subsection{Multilayer absorbers at normal light incidence}

A multilayer system with additional dielectric/metallic layers stacked on top of the resonator disc is studied. These discshaped, alternating metal and dielectric layers have thicknesses of 50 and $100 \mathrm{~nm}$, respectively. Note that an $n$-stack system represents the trilayer system with $n$ additional AlNAl stacks on top of the resonator. The following simulation parameters remain constant during the calculations: resonator radius and height, dielectric, bottom metal, and $\mathrm{SiO}_{2}$ substrate height are 500 and 50,115, 150, and $250 \mathrm{~nm}$, respectively. The absorption spectrum of a zero-stack system in Fig. 5a corresponds to the trilayer system at resonance frequency and serves here as a reference. It shows a nearunity absorption at a resonance wavelength of $5.19 \mu \mathrm{m}$ with a FWHM value of $650 \mathrm{~nm}$, and the respective maximum electric (magnetic) field value is $29.04 \mathrm{~V} \mathrm{~m}^{-1}\left(0.0728 \mathrm{~A} \mathrm{~m}^{-1}\right)$. Characteristic for the absorption behavior of a one-stack system is a spectral peak broadening with a FWHM value of $1050 \mathrm{~nm}$, which is due to the excitation of a second resonance at $4.85 \mu \mathrm{m}$ and its superposition with the absorption peak of the trilayer at $5.19 \mu \mathrm{m}$ (Fig. 5b). The absorption peak at $4.85 \mu \mathrm{m}$ results from a resonant coupling of the incident light mainly in the upper stack, which is indicated by the concentration of the electric and magnetic field in the upper circular dielectric layer (Fig. 5e). A steady increase of the wavelength leads to a gradual localization displacement of both fields, resulting in a dominant field concentration in the dielectric of the trilayer at the wavelength of $5.19 \mu \mathrm{m}$.
Compared to the plain trilayer system, both resonances feature a marginally weaker coupling of the electric and magnetic field, which leads to slightly lower absorption peaks. The difference in the excitation frequency arises from lateral geometrical differences. The reduced metallic area in the first stack might result in confined surface currents leading to two different effective permittivities and permeabilities. The calculated resonance peak in a two-stack system basically represents the superposition of three different resonances (Fig. 5c and f). The first, second, and third resonances appear at wavelengths of $4.83,4.97$, and $5.19 \mu \mathrm{m}$, respectively. Similar to the one-stack system, they are due to a main localization of the electric and magnetic field in the top, middle dielectric layer, and the dielectric of the trilayer (Fig. 5f). For comparison, the maximum electric and magnetic field magnitudes of the third resonance are comparable to those calculated in the plain trilayer and one-stack system. In contrast, due to a less efficient electromagnetic coupling the respective maximum electric (magnetic) field values of the first and second resonance are about $38 \%$ (30\%) lower, which lead to an overall nonlinear absorption decrease. Therefore, the peak of the third resonance is well pronounced compared to the first and second resonances, while the spectral peak broadening is still comparable to that observed in a one-stack system. Since the two disc-shaped stacks in the two-stack system hold the same geometry and consist of the same materials, one would expect the first two corresponding resonances to appear at the same wavelength. However, the decisive difference between them lies in their respective boundaries, which are defined by the adjacent layers surrounding the top and the middle stack. The top stack is sandwiched by air and the adjacent layers of the middle stack. Besides the interaction with the top stack, the middle stack is additionally influenced by the underlying trilayer, which finally leads to a slight resonance shift of about $0.14 \mu \mathrm{m}$. In contrast, the resonance wavelengths due to field localizations in the top dielectric layer remain almost constant for the one- and two-stack system (Fig. 5e and f). It should be noted at this point that, so far, only the geometrical parameters of the trilayer system have been optimized towards perfect absorption. Further optimizations by tuning the geometrical parameters of the disc-shaped stacks could minimize unwanted reflections of such multi-stack systems leading to highly efficient electromagnetic coupling at distinct wavelengths. This can result in multiple resonances with perfect absorption as exemplarily demonstrated in the following section for the one-stack system.

A modified design of the shown one-stack system was optimized toward the realization of a wavelength selective "perfect dual-band absorber" (Fig. 5d). The tailored radius of the top disc is $350 \mathrm{~nm}$, while the radius of the underlying dielectric and metallic layer is $575 \mathrm{~nm}$. Except for the radii, all other parameters remain identical. In accordance to Fig. 3a, two absorption peaks appear at a resonance wavelength of 3.0 and $5.0 \mu \mathrm{m}$ for absorber radius 350 and $575 \mathrm{~nm}$, respectively, although the disks are stacked in this case. The ab- 
sorption for both spectral bands reaches values very close to unity (> 99.9\%). The electric and magnetic resonance properties display similar characteristics to the unmodified zerostack metamaterial structure. As a consequence, a "perfect multiband absorber" can be designed by combining $n$-stack systems consisting of $n$ resonators of different size (Dayal and Ramakrishna, 2013).

\section{Conclusions}

Micron-sized composite structures consisting of aluminium and aluminium nitride layer stacks have been investigated as potential wavelength-selective absorbers for thermal detectors by numerical simulations. Electric and magnetic resonances are optimized by tuning the geometrical parameters of the absorber elements, resulting in near-unity absorption (PMA). Over $94 \%$ of the radiation intensity is absorbed at angles of incidence up to $40^{\circ}$. The circularly patterned resonator structures imply polarization-independent absorption. The integration of metamaterial structures in thermal detectors has been addressed by the implementation of typical passivation layers in the simulation model. No significant influences on the absorption properties could be observed. Higher-complexity metamaterials consisting of patterned multilayer resonators show a spectral broadening of the absorption peak due to additional resonances in close proximity to each other. Multilayer structures of differentsized resonators were designed, which can be utilized as multiband PMAs. The spectroscopic characterization of the presented metamaterial structures is part of current investigations.

Acknowledgements. The authors acknowledge funding from BMVIT, BMWFJ, and the Province of Lower Austria, COMET K2 Project, SF-Contract Area 5, project number C150401.

Edited by: Q. Sun

Reviewed by: two anonymous referees

\section{References}

Dayal, G. and Ramakrishna, S. A.: Design of highly absorbing metamaterials for Infrared frequencies, Opt. Express, 20, 1750317508, doi:10.1364/OE.20.017503, 2012.

Dayal, G. and Ramakrishna, S. A.: Design of multi-band metamaterial perfect absorbers with stacked metal-dielectric disks, J. Opt., 15, 055106, doi:10.1088/2040-8978/15/5/055106, 2013.

Kischkat, J., Peters, S., Gruska, B., Semtsiv, M., Chashnikova, M., Klinkmüller, M., Fedosenko, O., Machulik, S., Aleksandrova, A., Monastyrskyi, G., Flores, Y., and Masselink, W. T.: Mid-infrared optical properties of thin films of aluminum oxide, titanium dioxide, silicon dioxide, aluminum nitride, and silicon nitride, Appl. Optics, 51, 6789-6798, doi:10.1364/AO.51.006789, 2012.
Landy, N. I., Sajuyigbe, S., Mock, J. J., Smith, D. R., and Padilla, W. J.: Perfect metamaterial absorber, Phys. Rev. Lett., 100, 207402, doi:10.1103/PhysRevLett.100.207402, 2008.

Maier, T. and Brueckl, H.: Wavelength-tunable microbolometers with metamaterial absorbers, Opt. Lett., 34, 3012-3014, doi:10.1364/OL.34.003012, 2009.

Maier, T. and Brueckl, H.: Multispectral microbolometers for the midinfrared, Opt. Lett., 35, 3766-3768, doi:10.1364/OL.35.003766, 2010.

Ordal, M. A., Bell, R. J., Alexander, R. W., Long Jr., L. L., and Query, M. R.: Optical properties of fourteen metals in the infrared and far infrared, Appl. Optics, 24, 4493-4499, doi:10.1364/AO.24.004493, 1985.

Palik, E. D.: Handbook of Optical Constants of Solids, Academic Press, New York, USA, 1985.

Pendry, J. B., Holden, A. J., Stewart, W. J., and Youngs, I.: Extremely low frequency plasmons in metallic mesostructures, Phys. Rev. Lett., 76, 4773-4776, doi:10.1103/PhysRevLett.76.4773, 1996.

Pendry, J. B., Holden, A. J., Robbins, D. J., and Stewart, W. J.: Magnetism from conductors and enhanced nonlinear phenomena, IEEE T. Microw. Theory, 47, 2075-2084, doi:10.1109/22.798002, 1999.

Pimenov, A., Loidl, A., Gehrke, K., Moshnyaga, V., and Samwer, K.: Negative Refraction Observed in a Metallic Ferromagnet in the Gigahertz Frequency Range, Phys. Rev. Lett., 98, 197401, doi:10.1103/PhysRevLett.98.197401, 2007.

Schurig, D., Justice, J. J., Cummer, S. A., Pendry, J. B., Starr, A. F., and Smith, D. R.: Metamaterial electromagnetic cloak at microwave frequencies, Science, 314, 977-980, doi:10.1126/science.1133628, 2006.

Shelby, R. A., Smith, D., and Schultz, S.: Experimental verification of a negative index of refraction, Science, 292, 77-79, doi:10.1126/science.1058847, 2001.

Smith, D. R. and Pendry, J. B.: Homogenization of metamaterials by field averaging, J. Opt. Soc. Am. B., 23, 391-403, doi:10.1364/JOSAB.23.000391, 2006.

Tao, H., Landy, N. I., Bingham, C. M., Zhang, X., Averitt, R. D., and Padilla, W. J.: A metamaterial absorber for the terahertz regime: Design, fabrication and characterization, Opt. Express, 16, 71817188, doi:10.1364/OE.16.007181, 2008.

Veselago, V. G.: The electrodynamics of substances with simultaneously negative values of $\epsilon$ and $\mu$, Sov. Phys. Uspekhi, 10, 509514, doi:10.1070/PU1968v010n04ABEH003699, 1968.

Zeng, Y., Chen, H.-T., and Dalvit, D. A. R.: The role of magnetic dipoles and non-zero-order Bragg waves in metamaterial perfect absorbers, Opt. Express, 21, 3540-3546, doi:10.1364/OE.21.003540, 2013.

Zhao, Y., Zhu, C., Wang, S., Tian, J. Z., Yang, D. J., Chen, C. K., Cheng, H., and Hing, P.: Pulsed photothermal reflectance measurement of the thermal conductivity of sputtered aluminum nitride thin films, J. Appl. Phys., 96, 4563-4568, doi:10.1063/1.1785850, 2004. 